\title{
$\begin{array}{ll}\text { Research Square } & \text { Preprints are preliminary reports that have not undergone peer review. } \\ \text { They should not be considered conclusive, used to inform clinical practice, }\end{array}$ or referenced by the media as validated information.
}

\section{Incidental Gallbladder Cancer with the Laparoscopic Treatment in the MRI Era: An Experience at a Single Institution}

yong zhou ( $\sim$ zhouyong13616@sina.com )

yancheng city NO.1 people's hospital https://orcid.org/0000-0002-8220-1257

Wenzhang Zha

yancheng city NO.1 people's hospital

Xudong Wu

yancheng city NO.1 people's hospital

Rengen Fan

yancheng City N0.1 people's hospital

\section{Research}

Keywords: Incidental gallbladder cancer, Magnetic resonance imaging, Laparoscopic treatment, Intraoperative histological examination, Ultrasonography, Gallbladder diseases

Posted Date: March 18th, 2021

DOI: https://doi.org/10.21203/rs.3.rs-324781/v1

License: (c) (i) This work is licensed under a Creative Commons Attribution 4.0 International License. Read Full License 


\section{Abstract}

Background: Magnetic resonance imaging (MRI) has been widely applied to the preoperative diagnosis of gallbladder diseases, among which laparoscopic cholecystectomy plays a critical role. Incidental gallbladder cancer (IGBC) is an unexpected guest of the patients who underwent cholecystectomy, forcing them to receive reoperation for complete resection. This study aimed to share our experience in pre-operative MRI evaluation and laparoscopic management of the IGBCs.

Methods: Between January 2011 and January 2020, 7917 patients with gallbladder diseases and treated by laparoscopy were enrolled in this study.

Results: A total of 49 patients were diagnosed as IGBCs. The incidence of IGBCs in polypoid lesions, biliary pancreatitis, cholecystitis, cholecystocholedocholithiasis and gallbladder stones was $0.42 \%$, $1.19 \%, 0.62 \%, 1.20 \%$ and $0.49 \%$, respectively. Moreover, $73.5 \%$ and $59.2 \%$ of the IGBCs had unremarkable preoperative imaging during the ultrasonographic and MRI evaluation respectively. Also, 14 of the patients were diagnosed as gallbladder cancer (GBC) via intraoperative histological examination, and 11 received laparoscopic extensive resection after cholecystectomy.

Conclusion: The patients with biliary pancreatitis and cholecystocholedocholithiasis have a higher incidence of incidental gallbladder cancer. Preoperative MRI evaluation and intraoperative histological examination may help some incidental gallbladder cancers to achieve one-stage laparoscopic extensive resection.

\section{Background}

Gallbladder cancer (GBC), an aggressive and highly lethal disease, is the most frequent malignancy of the biliary tract. Laparoscopic cholecystectomy (LC) is considered as the gold-standard management for benign gallbladder disease. It is recommended for the tumors limited to the lamina propria (T1a) in the case of gallbladder cancer. Furthermore, open extended surgery should be performed for T1b, T2, and T3 lesions ${ }^{1-3}$. Only a third of gallbladder cancers are suspected preoperatively, while most of them are diagnosed incidentally by a pathological examination after surgery ${ }^{4}$. Therefore, to achieve complete resection, patients usually have to accept reoperation according to the cancer stage; while patients with T1a tumors are exceptions, who can be treated by simple cholecystectomy ${ }^{5}$.

With the development of laparoscopic skills, most of the gallbladder diseases have been treated by laparoscopic cholecystectomy; in the meantime, the incidence of IGBC has also risen, even up to $3 \%$ according to some reports ${ }^{6}$. Detecting incidental GBC preoperatively in clinical practice is hard since no mass can be observed on stone diseases; while cholecystectomy is generally performed to presumed benign polypoid lesions. Abdominal ultrasound (US) and computed tomography (CT) are commonly used in the pre-operational assessment of gallbladder diseases. Such imaging signs as suspicious lesions and irregularity of the gallbladder wall may provide useful information for preoperative diagnosis of incidental 
GBC. Nonetheless, none of them has been widely accepted clinically due to their unsatisfactory sensitivity and specificity ${ }^{7}$. In our hospital, applying US and MRI to gallbladder diseases for pre-operational evaluation is a common practice. This study aimed to share our experience in pre-MRI evaluation and laparoscopic management of the IGBCs.

\section{Methods}

\section{Patients}

This study was reviewed and approved by our institutional review board.

A retrospective study was performed on 7917 patients, who were referred to our institution from January 2011 to January 2020 for the management of gallbladder diseases. The preoperative diagnosis of these patients was as follows: cholecystitis (325 cases), gallbladder polyps (721 cases), biliary pancreatitis (252 cases) and gallbladder stones with (1164 cases) or without choledocholithiasis (5455 cases). This study was reviewed and approved by our institutional review board.

\section{Treatment And Data Collection}

Preoperative ultrasonography and magnetic resonance imaging/ magnetic resonance cholangiopancreatography (MRI/MRCP) were performed to determine the concomitant choledocholithiasis and to exclude gallbladder cancer in the patients. In addition, enhanced computer tomography was performed on preoperative suspicious cases to exclude the malignancy (Table 1).

Table 1

Baseline characteristics of the patients

\begin{tabular}{|ll|}
\hline Parameter & Number (\%) \\
\hline SEX & \\
\hline Female & $4354(55)$ \\
\hline Male & $3563(45)$ \\
\hline Preoperative diagnosis & \\
\hline Cholecystitis & $325(4.1)$ \\
\hline Gallbladder polyps & $721(9.1)$ \\
\hline Gallbladder stones & $5455(68.9)$ \\
\hline Cholecystocholedocholithiasis & $1164(14.7)$ \\
\hline Biliary pancreatitis & $252(3.2)$ \\
\hline
\end{tabular}


The patients with acute pancreatitis, cholangitis, and jaundice were treated before the operation. Those with concomitant choledocholithiasis received intraoperative bile duct exploration after the laparoscopic cholecystectomy or ERCP/EST before the laparoscopic cholecystectomy. All postoperative histological reports were reviewed and 49 patients were diagnosed with IGBCs. Eventually, their preoperative radiology findings and clinicopathological characteristics were recorded and analyzed. The patient enrollment and treatment algorithm are present in Fig. 1.

\section{Results}

\section{General information about the IGBC patients}

Of the 7917 patients, 3 patients with polypoid lesions, 3 with biliary pancreatitis, 2 with cholecystitis, 14 with cholecystocholedocholithiasis and 27 with gallbladder stone diseases were diagnosed with IGBCs intraoperatively and(or) postoperatively. Among them, 13 were males and 36 were females, with the age range from 48 to 89 (Table 2).

Table 2

Baseline characteristics of the IGBCs

\begin{tabular}{|ll|}
\hline Parameter & Number (\%) \\
\hline Fex & \\
male & $36(73.5)$ \\
\hline Age & $13(26.5)$ \\
$\leq 60$ & \\
$>60$ & $16(32.7)$ \\
\hline Presenting complaints & $33(67.3)$ \\
Abdominal pain & \\
Jaundice & $39(79.9)$ \\
NONE & $3(6.1)$ \\
\hline Preoperative diagnosis & $7(14.0)$ \\
Cholecystitis & \\
Gallbladder polyps & $2(4.1)$ \\
Gallbladder stones & $3(6.1)$ \\
Biliary pancreatitis & $27(55.1)$ \\
Cholecystocholedocholithiasis & $3(6.1)$ \\
\hline
\end{tabular}




\section{Incidence Of Igbcs In Different Gallbladder Diseases}

The incidence of IGBCs in polypoid lesions, biliary pancreatitis, cholecystitis, cholecystocholedocholithiasis and gallbladder stones was calculated as $0.42 \%$ ( 3 to 721 ), $1.19 \%$ (3 to 252), $0.62 \%$ ( 2 to 325 ), $1.20 \%$ (14 to 1164 ) and $0.49 \%$ (27 to 5455 ), respectively (Table 3 ).

Table 3

Incidence of IGBCs in different gallbladder diseases

\begin{tabular}{|ll|}
\hline Gallbladder diseases & Number (\%) \\
\hline Cholecystitis & $2(0.62)$ \\
\hline Gallbladder polyps & $3(0.42)$ \\
\hline Gallbladder stones & $27(0.49)$ \\
\hline Cholecystocholedocholithiasis & $14(1.20)$ \\
\hline Biliary pancreatitis & $3(1.19)$ \\
\hline
\end{tabular}

\section{Preoperative Radiology Of The Igbcs}

All the IGBCs received ultrasonographic and MRI evaluation preoperatively. Diffuse thickening of the gallbladder with or without suspicion of malignancy and focal thickening of the gallbladder was observed in 2, 5 and 4 patients respectively through ultrasound examination. Furthermore, the suspicious lesion was observed in 2 patients. And $73.5 \%$ of the patients had unremarkable preoperative imaging during the ultrasonographic evaluation (Table 4). However, during the MRI examination, diffuse thickening of the gallbladder with or without suspicion of malignancy and focal thickening of the gallbladder were observed in 6, 3 and 8 patients respectively. Furthermore, 3 patients displayed the suspicious lesion. Overall, $59.2 \%$ of the patients had unremarkable preoperative MRI imaging (Table 4).

Table 4

Preoperative radiology of the IGBCs

\begin{tabular}{|lll|}
\hline Radiology Findings & Ultrasonography (Number,\%) & $\begin{array}{l}\text { MRI } \\
\text { (Number,\%) }\end{array}$ \\
\hline Diffuse suspicious thickening & $2(4.1)$ & $6(12.2)$ \\
\hline Diffuse thickening not suspicious of malignancy & $5(10.2)$ & $3(6.1)$ \\
\hline Focal Thickening & $4(8.2)$ & $8(16.3)$ \\
\hline Suspicious lesion & $2(4.1)$ & $3(6.1)$ \\
\hline Non specific & $36(73.5)$ & $29(59.2)$ \\
\hline
\end{tabular}




\section{Surgical Information And Postoperative Pathology Of The Igbcs}

Additionally, 47 patients underwent laparoscopic cholecystectomy or the following common bile duct exploration; 2 received only laparoscopic exploration due to peritoneal dissemination; 15 received intraoperative histological examination, among whom 14 were diagnosed with GBC intraoperatively and 11 received laparoscopic extensive resection after the cholecystectomy. Of the 33 cases diagnosed after LC, 12 received laparoscopic extensive resection and the rest received open extensive resection four weeks later. According to the pathological TNM classification system, the tumor stages of the IGBCs were pT1a (10 cases), pT1b (14 cases), pT2 (16 cases), pT3 (7 cases) and pT4 (2 cases). Regarding the pathological characteristics of tumors, one case was squamous cell carcinomas, two were papillary carcinoma, one was adenosquamous carcinoma and 45 were adenocarcinomas. Further, among the 45 adenocarcinomas patients, 13 were poorly differentiated, 13 were moderately differentiated, and 19 were well differentiated. The tumor stages and histopathological characteristics of IGBCs are listed in Table 5.

Table 5

Tumor stage and histopathological type of incidental gallbladder cancer

\begin{tabular}{|llll|}
\hline & Intra-operative diagnosis & Post-operative diagnosis & Total \\
\hline Tumor stage & 3 & & \\
\hline T1a & 2 & 7 & 10 \\
\hline T1b & 6 & 12 & 14 \\
\hline T2 & 3 & 10 & 16 \\
\hline T3 & 2 & 4 & 7 \\
\hline T4 & & 0 & 2 \\
\hline Histopathological type & 0 & & 1 \\
\hline Squamous cell carcinomas & 0 & 1 & 2 \\
\hline Papillary carcinoma & 0 & 2 & 1 \\
\hline Adenosquamous carcinoma & 0 & 1 & 45 \\
\hline Adenocarcinomas & 16 & 29 & \\
\hline
\end{tabular}

\section{Discussion}

Incidental cancer of gall bladder refers to malignancy that is diagnosed through intra-operative or postoperative histological examination for presumed benign gallbladder disease. Traditionally, IGBCs have been considered to be an early-stage tumors with more favorable pathologic characteristics compared to non-incidentally diagnosed $\mathrm{GBCs}^{8}$. However, recent studies revealed that stage-matched outcomes for 
IGBCs were significantly worse than those operated with initially diagnosed GBCs. Thus, accurate preoperative diagnosis becomes imperative, which may reduce long-term morbidity and mortality of IGBCs $^{9-11}$.

Ultrasonography, CT and MRI are widely performed for the preoperative evaluation of the gallbladder diseases. Ultrasonography as a common tool for gallbladder diseases depends largely on the operator's skills ${ }^{12}$, which is characterized with low accuracy. CT and MRI are able to capture more accurate and objective features, which help distinguish the benign from the malignant ${ }^{13}$. MRI shares the same sensitivity and specificity of detection with CT ${ }^{14}$. As shown in our study, $40.8 \%$ of the IGBCs had preoperative imaging findings during the MRI evaluation, such as diffuse thickening of the gallbladder with or without suspicion of malignancy, focal thickening of the gallbladder and suspicious lesions. Furthermore, after the macroscopic examination of the specimens, 15 of them (75\%) received intraoperative histological examination. Therefore, it was speculated that macroscopic re-evaluation could improve the specificity of the MRI examination.

Laparoscopic cholecystectomy is the first choice for benign gallbladder diseases and even suspicious gallbladder cancers in some institutes. IGBCs are diagnosed more commonly among patients with laparoscopy cholecystectomy than those with open cholecystectomy. A recent study exposed that the laparoscopic and open cholecystectomy had little influence on the prognosis of incidental gallbladder cancer $^{15}$. Even for the IGBCs' re-operation, laparoscopic management could be considered as a valid alternative in the extensive resection of IGBCs ${ }^{16}$. As shown in our study, 47 IGBC patents experienced laparoscopic cholecystectomy and 11 patients received laparoscopic extensive resection following the cholecystectomy after intra-operative diagnosis. Furthermore, 12 with T1b stage tumors received laparoscopic extensive resection 4 weeks later after the first LC. Compared to laparoscopic reoperation, one-stage laparoscopic extensive resection can be more easily handled. Therefore, accurate pre- or intraoperative diagnosis plays an important role in the management of IGBCs.

Nomograms have been increasingly used as diagnostic tools to distinguish GBC from gallbladder diseases ${ }^{17-18}$. Nevertheless, these tools may be not valuable in IGBCs, since many of them have no remarkable preoperative signs. Radiological findings such as thickening of the gallbladder wall and/or polyps can be the subtle signs of malignancy for gallbladder diseases ${ }^{19-20}$. In our study, $26.5 \%$ and $40.8 \%$ of the patients had subtle preoperative imaging during the ultrasonographic and MRI evaluation, respectively. Although preoperative contrast-enhanced CT excluded the malignancy from these suspicious cases, macroscopic re-evaluation was still performed during the operation. Notably, 15 cases received frozen section examination, among whom, 14 were diagnosed with GBCs intra-operatively. Therefore, we conclude that the subtle MRI findings can provide useful information for the diagnosis of IGBC. The combination between macroscopic re-evaluation and intraoperative frozen pathological examination will increase the accuracy and allow some incidental gallbladder cancers to achieve onestage laparoscopic extensive resection, especially for the cases confined to the gallbladder wall. 


\section{Conclusions}

In conclusion, we found that the patients with biliary pancreatitis and cholecystocholedocholithiasis were associated with a higher incidence of incidental gallbladder cancer. Laparoscopic management could play an important role in the re-operation of IGBCs. Preoperative MRI evaluation and intraoperative histological examination may help some incidental gallbladder cancers to achieve one-stage laparoscopic extensive resection.

\section{Abbreviations}

MRI Magnetic resonance imaging

IGBC Incidental gallbladder cancer

US Abdominal ultrasound

CT Computed tomography

GBC Gallbladder cancer

LC Laparoscopic cholecystectomy

TNM Tumor-Node-Metastasis

\section{Declarations}

\section{Authors' contributions}

Yong Zhou, Wen-zhang Zha and Xu-dong Wu provided oversight for the project. Wen-zhang Zha, Yong Zhou, and Ren-gen Fan performed the operations. Yong Zhou and Ren-Gen Fan contributed to data collections, data analysis and writing.

\section{Acknowledgements}

None.

\section{Funding}

The study was supported by the Jiangsu Provincial Young Talents Program of Medicine (Grant No. QNRC2016471), Scientific Research Project of Jiangsu Provincial Health Commission (Grant No. M2020100) and Yancheng Medical Science Development Foundation (Grant No. YK2017006).

\section{Conflict of interest}

The authors declare no conflicts of interest. 
Availability of data and materials

The data that support the findings of this study are available from the corresponding author on reasonable request.

\section{Ethics approval and consent}

All procedures performed in studies involving human participants were in accordance with the ethical standards of the institutional and/or national research committee and with the 1964 Helsinki declaration and its later amendments or comparable ethical standards. This study is approved by Ethics Committee of Yancheng City NO.1 People's Hospital.

\section{Consent for publication}

It is unavailable.

\section{References}

1. Lee SE, Jang JY, Lim CS, Kang MJ, Kim SW. Systematic review on the surgical treatment for T1 gallbladder cancer. World J Gastroenterol. 2011;17:174-80.

2. Wakai T, Shirai Y, Yokoyama N, Nagakura S, Watanabe H, Hatakeyama K. Early gallbladder carcinoma does not warrant radical resection. Br J Surg. 2001;88:675-8.

3. Goetze TO, Paolucci V. Benefits of reoperation of T2 and more advanced incidental gallbladder carcinoma: analysis of the German Registry. Ann Surg. 2008;247:104-8.

4. Goetze T, Paolucci V. Does laparoscopy worsen the prognosis for incidental gallbladder cancer? Surg Endosc. 2006;20(2):286-93.

5. Zaidi MY, Maithel SK. Updates on gallbladder cancer management. Curr Oncol Rep. 2018;20:21.

6. Toyonaga T, Chijiiwa K, Nakano K, Noshiro H, Yamaguchi K, Sada M, et al. Completion radical surgery after cholecystectomy for accidentally undiagnosed gallbladder carcinoma. World J Surg. 2003;27:266-71.

7. Isambert M, Leux C, Métairie S, Paineau J. Incidentally- discovered gallbladder cancer: When, why and which reoperation? J Visc Surg. 2011;148:e77-84.

8. Ethun CG, Le N, Lopez-Aguiar AG, Pawlik TM, Poultsides G, Tran T, et al. Pathologic and Prognostic Implications of Incidental versus Nonincidental Gallbladder Cancer: A 10-Institution Study from the United States Extrahepatic Biliary Malignancy Consortium. Am Surg. 2017;83:679-86.

9. Steinert R, Nestler G, Sagynaliev E, Müller J, Lippert H, Reymond MA. Laparoscopic cholecystectomy and gallbladder cancer. J Surg Oncol. 2006;93:682-9.

10. Kim JH, Kim WH, Kim JH, Yoo BM, Kim MW. Unsuspected gallbladder cancer diagnosed after laparoscopic cholecystectomy: focus on acute cholecystitis. World J Surg. 2010;34:114-20. 
11. Clemente G, Nuzzo G, De Rose AM, Giovannini I, La Torre G, Ardito F, et al. Unexpected gallbladder cancer after laparoscopic cholecystectomy for acute cholecystitis: a worrisome picture. J Gastrointest Surg. 2012;16:1462-8.

12. Hammad AY, Miura JT, Turaga KK, Johnston FM, Hohenwalter MD, Gamblin TC. A literature review of radiological findings to guide the diagosis of gallbladder adenomyomatosis. HPB (Oxford). 2016;18:129-35.

13. Chen M, Cao J, Bai Y, Tong C, Lin J, Jindal V, et al. Development and Validation of a Nomogram for Early Detection of Malignant Gallbladder Lesions. Clin TransI Gastroenterol. 2019;10(10):e00098.

14. Hu Y, Jia D, Xu Z, Wang J, Cai H, Yang W, et al. Value of diffusion-weighted MRI in differentiation between benign and malignant polypoid gallbladder lesions. Zhonghua Yi Xue Za Zhi. 2015;95:3201-4.

15. Goetze TO, Paolucci V. Prognosis of incidental gallbladder carcinoma is not influenced by the primary access technique: analysis of 837 incidental gallbladder carcinomas in the German Registry. Surg Endosc. 2013;27:2821-8.

16. de Aretxabala X, Oppliger F, Solano N, Rencoret G, Vivanco M, Carvajal D, et al. Laparoscopic management of incidental gallbladder cancer. Surg Endosc. 2018;32(10):4251-5.

17. Jang JY, Park T, Lee S, Kim Y, Lee SY, Kim SW, et al. Proposed nomogram predicting the individual risk of malignancy in the patients with branch duct type intraductal papillary mucinous neoplasms of the pancreas. Ann Surg. 2017;266:1062-8.

18. Freedman AN, Seminara D, Gail MH, Hartge P, Colditz GA, Ballard-Barbash R, et al. Cancer risk prediction models: A workshop on development, evaluation, and application. J Natl Cancer Inst. 2005;97:715-23.

19. Rammohan A, Cherukuri SD, Sathyanesan J, Palaniappan R, Govindan M. Incidental gall bladder cancers: Are they truly incidental? World J Gastrointest Oncol. 2014;6(12):441-3.

20. Cha SY, Kim YK, Min JH, Lee J, Cha DI, Lee SJ. Usefulness of noncontrast MRI in differentiation between gallbladder carcinoma and benign conditions manifesting as focal mild wall thickening. Clin Imaging. 2019;54:63-70.

\section{Figures}




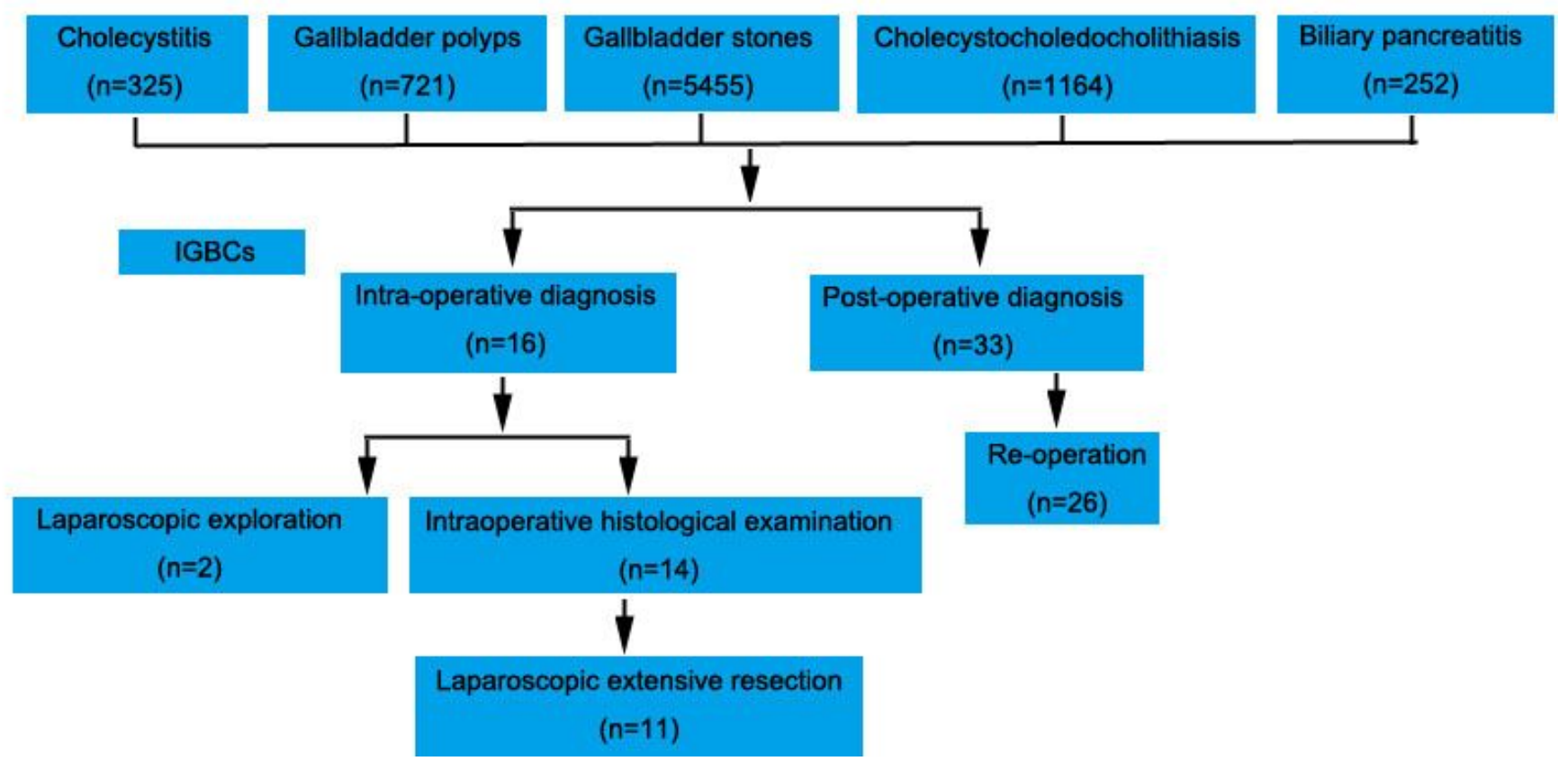

\section{Figure 1}

Flow chart of patient enrollment and treatment algorithm. 